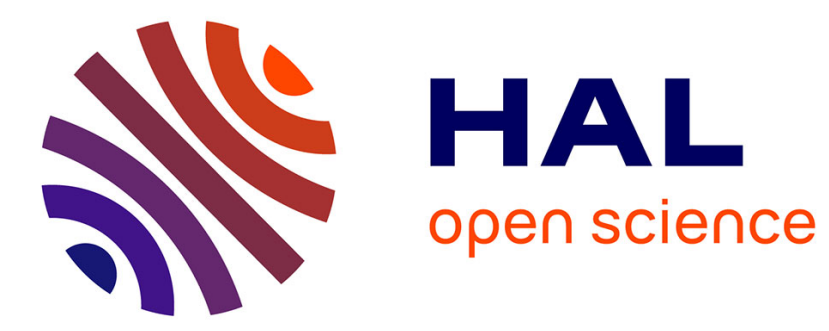

\title{
A Sensing and Computational Framework for Estimating the Seismic Velocities of Rocks Interacting With the Drill Bit
}

Jean Auriol, Nasser Kazemi, Roman J. Shor, Kristopher Innanen, Ian Gates

\section{To cite this version:}

Jean Auriol, Nasser Kazemi, Roman J. Shor, Kristopher Innanen, Ian Gates. A Sensing and Computational Framework for Estimating the Seismic Velocities of Rocks Interacting With the Drill Bit. IEEE Transactions on Geoscience and Remote Sensing, In press, 10.1109/TGRS.2019.2950257 . hal02462287

\section{HAL Id: hal-02462287 \\ https://hal.science/hal-02462287}

Submitted on 31 Jan 2020

HAL is a multi-disciplinary open access archive for the deposit and dissemination of scientific research documents, whether they are published or not. The documents may come from teaching and research institutions in France or abroad, or from public or private research centers.
L'archive ouverte pluridisciplinaire HAL, est destinée au dépôt et à la diffusion de documents scientifiques de niveau recherche, publiés ou non, émanant des établissements d'enseignement et de recherche français ou étrangers, des laboratoires publics ou privés. 


\title{
A sensing and computational framework for estimating the seismic velocities of rocks interacting with the drill-bit
}

\author{
Jean Auriol* ${ }^{*}$, Nasser Kazemi ${ }^{* \dagger} \ddagger$, Roman J. Shor* ${ }^{*}$, Kristopher A. Innanen ${ }^{\dagger}$, Ian D. Gates* \\ * Department of Chemical and Petroleum Engineering, University of Calgary \\ $\dagger$ Department of Geoscience, CREWES, University of Calgary \\ $\ddagger$ Integrated Drilling Research Laboratory, University of Calgary
}

\begin{abstract}
We have developed a sensing and computational framework to estimate seismic velocities of rocks interacting with the drill-bit during the drilling process. The performance of drilling depends on our knowledge of the subsurface. The interaction between the drill-bit and rock can introduce severe vibrations in the drill-string and result in safety and performance issues. However, we can use seismic waves radiated from drill-bit-rock interactions to determine seismic velocities of the rocks interacting with the drill-bit. Our approach consists of a distributed (wave equation) representation of the dynamics of the drill-string for which we show (using Riemann's invariants and a backstepping approach) that it is possible to express the force-on-bit as a function of the top-drive force and the topdrive velocity, without requiring explicit information about the subsurface properties. We also show that seismic waves generated by drill-bit-rock interaction can be modelled as functions of the force-on-bit and of rock velocities. The rock velocity independent formulation of the force-on-bit, along with modelling of the seismic waves generated by drill-bit-rock interaction as a function of force-on-bit and rock velocities allow us to estimate seismic velocities of rocks interacting with the drill-bit. We use the alternating minimization algorithm to estimate the velocities. Numerical examples on simulated data are indicators of the validity of the approach. The proposed methodology is the first step towards a subsurface-aware drilling system.
\end{abstract}

\section{INTRODUCTION}

Optimizing the drilling process requires that subsurface material properties be accurately known. Drill-bit-rock interaction can cause severe vibrations in the drilling system and result in an inefficient Rate Of Penetration (ROP). It may also raise safety issues. Control mechanisms may be used to reduce these vibrations in the system [1], [2]. However, such control mechanisms often depend on the rock properties that the system is drilling into. Hence, having access to the accurate subsurface information in near real-time will allow us to design an efficient drilling system. Here, we only focus on estimating the seismic velocities of rocks interacting with the drill-bit in the drilling process. To do so, the first step is to understand the drilling mechanism.

The drilling of an oil well consists of creating a borehole up to several thousand meters deep into the ground until an oil reservoir is reached. The mechanical part of a drilling system is made of three components: the rotating mechanism

Corresponding author: J. Auriol (email: jean.auriol@ucalgary.ca). (usually a rotary table or a top drive), the drill-string and the Bottom Hole Assembly (BHA). The dynamical behaviour of drill-strings is complex and involves many dynamic phenomena (vibrations, bending and twisting quasi-static motion, bit-rock interactions [3], [4]). To describe the dynamics of interest, distributed parameter models have been proposed in the literature [5], [6]. In this class of models, the axial and torsional efforts can be modeled by a set of hyperbolic Partial Differential Equations (PDEs). For this class of representation, special attention must be paid to the boundary conditions and in particular to the bit-rock interaction law at the downhole boundary [7]-[9] that depends on the drilled rock properties. The drill-string interaction with the borehole gives rise to a wide variety of non-desired oscillations [10]-[12] which have been extensively studied. Among them, torsional oscillations known as stick-slip are considered to be one of the most prevalent vibrations. These stick-slip oscillations are characterized by a series of stopping - "sticking" - and releasing - "slipping" - events of the bit. These vibrations may lead to a reduction of the ROP, cause fatigue on the equipment and wellbore instability. They can also lead to premature failure of the bit and may cause catastrophic failures, or, at least, wear to expensive components of the drill-string [13]. Such oscillations can be the consequence of specific downhole conditions (rock composition or small borehole diameter). Numerous models assume that stick-slip is a consequence of the non-linear frictional force actuating at the bit by contact with the rock [14], [15]. More precisely, in these models, the stick-slip phenomenon is assumed to be related to the velocity-weakening of the frictional force at the bit (Stribecklike effect) associated with typical dry friction profiles (static friction and dynamic friction) [3], [16]. However, the bit-rock interaction is not the only cause of stick-slip; for instance, it would not explain the occurrence of such oscillations in the case of bit off bottom [17]-[19].

A clear understanding of drill-string dynamics appears to be crucial to control these vibrations and to obtain an improvement of the performance of drilling systems (ROP), preventing damage and reducing safety risks. Moreover, as the cost of drilling a well is in large part determined by the time it takes, strategies for reducing non-productive time and increasing ROP are sought. Different models of friction at the contact point between the cutting device and the drilling 
surface have been proposed in the literature [6]. These models usually depend on the nature of the drilled rock (dependence of the drill-string dynamic response on bit-rock interaction and rock properties were verified in [20]). They can later be used to express the downhole boundary condition of the distributed parameter models previously mentioned, such a boundary condition being for instance found by expressing the weight-on-bit, which are the result of cutting forces and friction forces. Estimating the seismic velocities of rocks while drilling is not an easy task as downhole sensors are expensive and may raise potential technical risks. A viable option is to remotely record the seismic energy generated by the drillbit rock interactions and use the recorded measurements to estimate the seismic velocities of the rocks interacting with the drill-bit.

Since the 1960s, recording and interpreting the seismic energy generated by the drill-bit-rock interaction has been an active area of research [21]-[23]. This methodology is called seismic while drilling (SWD). The SWD data are rich in information, and, provided that we understand the nonimpulsive and correlative source signature of the drill-bit rock interaction, they can enhance our knowledge of the subsurface materials. In recent years, due to the advances in modelling the radiation patterns of the drill-bit rock interaction and increased computational power in the field, new methodologies have been developed [24]-[31]. Here, we make use of such data to estimate the seismic velocities of rocks interacting with the drill-bit.

In this paper, we present a new algorithm for estimating the seismic velocities of rocks interacting with the drillbit. The proposed algorithm uses far-field radial and angular displacements (obtained by using seismic sensors) combined with the measurements of the drill-string top-drive force and top-drive velocity. Our methodology only requires knowledge of the physical properties of the drilling system such as its Young's modulus, pipe density, etc, but does not require any knowledge about the subsurface properties. Employing a distributed axial dynamics of the drill-string, we show that the force exerted on the bit can be expressed as a function of the top-drive force and top-drive velocity (which are measured) without depending on the downhole boundary condition or on the subsurface. Combining this relationship with a seismicwhile-drilling approach, we can express the far-field radial and angular displacement as a function of the force-on-bit and rock velocity, the only unknown parameters now being the seismic velocities of the rock. We implement alternating minimization approach to estimate the seismic velocities of the rocks interacting with the drill-bit in near real-time. This is the first step towards a subsurface-aware drilling system.

The paper is organized as follows. In Section II, we derive a distributed model to describe the axial dynamics of the drill-string. Seismic-while-drilling techniques are described in Section III. In Section IV, we derive the Riemann invariants associated with the distributed model of the drill-string. Then, using a backstepping approach, we express the drill-bit axial force as a function of the top-drive force and velocity. This relationship is used in Section V to develop a new algorithm that estimates the seismic velocities of the rocks ahead of
TABLE I

NOMENCLATURE

\begin{tabular}{cl} 
Parameters \\
\hline$E$ & pipe Young's modulus \\
$\rho$ & pipe mass density \\
$c_{\xi}$ & axial wave velocity \\
$k_{\xi}$ & viscous shear stesses \\
$A$ & cross-sectional area of the drill-string \\
$L$ & drill-string length \\
$M_{b}$ & mass of the lumped BHA \\
$\omega_{b i t}$ & bit angular velocity \\
$a$ & bit radius \\
$\zeta$ & characterization of the cutting force \\
$w_{f}$ & friction weight \\
$\epsilon$ & intrinsic specific energy of the rock \\
$\rho_{f}$ & density of the rock \\
$V_{p}$ & rock compressional velocity \\
$V_{s}$ & rock shear velocity \\
Dependent variables \\
\hline$\xi$ & drill-string axial displacement \\
$w$ & drill-string axial force \\
$v$ & drill-string axial velocity \\
$u, z$ & drill-string Riemann invariants \\
$w_{0}$ & weight on the drill-string \\
$U_{r}$ & P-wave radiation \\
$U_{\phi}$ & S-wave radiation \\
Independent variables \\
\hline$t$ & time \\
$x$ & position relative to top of drill-string \\
\end{tabular}

the drill-bit. Some simulation results are given Section VI. A discussion about future work is given in Section VII. Finally, some concluding remarks are given in Section VIII.

In what follows, given a set $\Omega \subseteq \mathbb{R}$, its characteristic function will be denoted by

$$
\mathbb{1}_{\Omega}(\theta)= \begin{cases}1 & \text { if } \theta \in \Omega \\ 0 & \text { otherwise. }\end{cases}
$$

\section{DRILL-STRING MODEL}

In this section we present the model under consideration to describe the mechanical dynamics of the drilling system.

\section{A. Distributed Axial dynamics of the drill-string}

Let us consider a vertical well, as pictured in Figure 1. The drilling system consists of three parts: the rotary table, the drill-string and the drill-bit. We consider the case of a rollercone drill-bit.

The rotary table sets the drill-string into a rotary motion around its main axis and the dynamics of interest can be derived by assuming elastic deformations and using equations of continuity and state. More precisely, a distributed model has been proposed in [5], [32] to describe the evolution of the axial displacement $\xi(t, x)$ of the drill-string which is a function of $(t, x)$ evolving in $\{(t, x) \mid 0<t<T, x \in[0, L]\}$ (where $L$ is the total length of the drill-string and $T$ a positive time). The axial motion satisfies the following wave Partial Differential Equation

$$
\frac{\partial^{2} \xi}{\partial t^{2}}(t, x)-c_{\xi}^{2} \frac{\partial^{2} \xi}{\partial x^{2}}(t, x)=-k_{\xi} \frac{\partial \xi}{\partial t}(t, x),
$$

where $c_{\xi}=\sqrt{\frac{E}{\rho}}, \rho$ being the pipe mass density $E$ its Young's modulus and $k_{\xi}$ is a damping coefficient representing the 


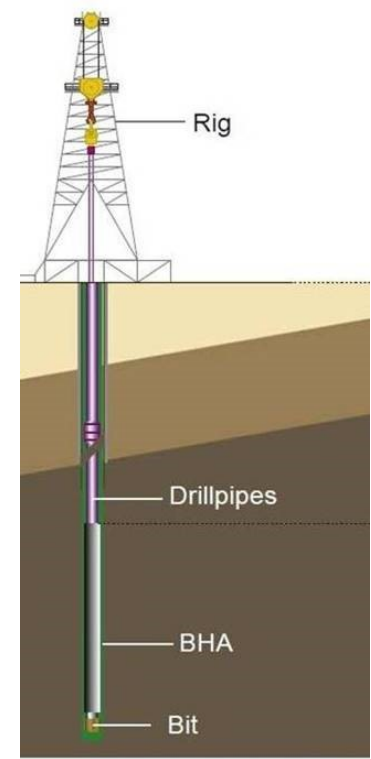

Fig. 1. Schematic representation of a drilling system (modified from [14])

viscous shear stresses acting on the pipe. The axial force associated to $\xi$ can be found from the strain, given as the local relative compression:

$$
w(t, x)=A E \frac{(\xi(t, x)-\xi(t, x+d x))}{d x},
$$

$A$ being the cross-sectional area of the drill-string and $d x \rightarrow 0$ the infinitesimal axial position increment. The axial velocity satisfies

$$
v(t, x)=\frac{\partial \xi(t, x)}{\partial t} .
$$

It can easily be shown from (1) that the axial force and velocity satisfy the following set of PDEs

$$
\begin{aligned}
& \frac{\partial w(t, x)}{\partial t}+A E \frac{\partial v(t, x)}{\partial x}=0, \\
& \frac{\partial v(t, x)}{\partial t}+\frac{1}{A \rho} \frac{\partial w(t, x)}{\partial x}=-k_{\xi} v(t, x) .
\end{aligned}
$$

The topside weight on the drill-string, $w(t, 0)$, corresponds to the system actuation. The downhole boundary condition at $x=$ $L$ is obtained from a force balance on the lumped BottomHole Assembly (BHA). These two boundary conditions will be discussed in the following.

\section{B. Topside boundary condition}

In the considered drilling system, the drill-string is connected at the top to the top-drive suspended over the drill floor by the traveling block. This block is connected by several drill lines with one attached to the deadline anchor and the other being spooled on a drum controlled by AC induction motors [33]. Thus, we can assume that the operator control the weight on the drill-string $w_{0}(t)=w(t, 0)$. This yields

$$
-E A \frac{\partial \xi(t, x)}{\partial x}=w_{0}(t),
$$

where $w_{0}$ is considered as an input.

\section{Downhole boundary condition: bit-rock interaction}

The lowermost section of the drill-string is typically made up of drill collars which may have a great impact on the drill-string dynamic due to their added inertia. In particular, the transition from pipes to collars in the drill-string will cause reflections in the traveling waves due to the change in characteristic line impedance [34]. Rigorously speaking, we should write a new wave PDE for the Bottom-Hole Assembly (BHA). This PDE would be analogous to (1) but using the BHA density and Young's modulus. However, as the length of the BHA $(\approx 200 \mathrm{~m})$ is much smaller than the one of the drill-string $(\approx 2000 \mathrm{~m})$, its effect can be lumped into an ODE coupled with the drill-string [5]. Thus, the downhole boundary condition at $x=L$ can be obtained from a force balance on the lumped BHA. This yields

$$
M_{b} \frac{\partial v}{\partial t}(t, L)=-w_{b}(v(t, L), w(t, L))+w(t, L),
$$

where $M_{b}$ is the mass of the lumped BHA and $w_{b}(\cdot, \cdot)$ the force acting from the rock on the BHA through the drilling bit, known as the weight on bit. From [32], [33], the weight on bit can be related to the bit velocity by considering the combined depth of cut per revolution [35]. More precisely, the combined depth of cut per revolution $d(t)$ satisfy the following equation,

$$
d(t)=\frac{v(t, L)}{\omega_{\text {bit }}},
$$

where the bit angular velocity $\omega_{\text {bit }}$ is assumed constant here. The cutting force is finally expressed as

$$
w_{b}(v(t, L), w(t, L))=w_{f}+K d(t)=w_{f}+a \zeta \epsilon d(t),
$$

where $w_{f}$ is a friction weight independent of the bit velocity (and therefore constant) while $K=a \zeta \epsilon$, with $a$ being the bit radius, $\zeta$ a characterization of the cutting angle and $\epsilon$ the intrinsic specific energy of the rock [35], [36]. Thus, the boundary condition (6) rewrites as

$$
M_{b} \frac{\partial^{2} \xi}{\partial t^{2}}(t, L)=-\frac{a \zeta \epsilon}{\omega_{\mathrm{bit}}} \frac{\partial \xi(t, L)}{\partial t}-w_{f}-E A \frac{\partial \xi(t, L)}{\partial x} .
$$

Due to the complexity of this boundary condition, controlling the weight on the drill-string to achieve optimal drilling performance is not an easy task. It has to be updated in realtime to adapt to the changing operating conditions (different type of rocks for instance). To do so, classical control procedures rely on topside drilling data and either assume known the nature of the drilled rock or use simple PID controllers. This explains why the knowledge of the nature of the subsurface could lead to an improvement in the performance of the control mechanism and thus optimize ROP while drilling.

\section{SEISMIC WHILE DRILLING}

Each layer in the subsurface is considered to be a homogenous and isotropic medium. In this model, each layer is recognized by its seismic velocities. The seismic velocities depend on Lamé parameters. In the drilling process, the impact of drill-bit on the rock generates vibrations. These vibrations vary depending on the drilling configurations and the Lamé parameters of the drilled rock. For example, the pure axial 
motion of the drill-bit in vertical direction generates pressure wavefield in the direction of motion and SV (Shear wave vertical component) wavefield in the direction perpendicular to the drill-bit motion. The radiation patterns of such motion are depicted in Figure 2. The vertical only motion of drill-bit does not generate SH (Shear wave horizontal component) wavefield. However, if the drill-bit rotates in the drilling process the impact of rotating motion on the layer can generate $\mathrm{SH}$ waves. These radiated seismic wave energies can be recorded by the receivers at the surface. The recorded seismic while drilling data provides vital information about the subsurface materials provided that we understand the behavior of the seismic radiation patterns of the drill-bit rock interaction.

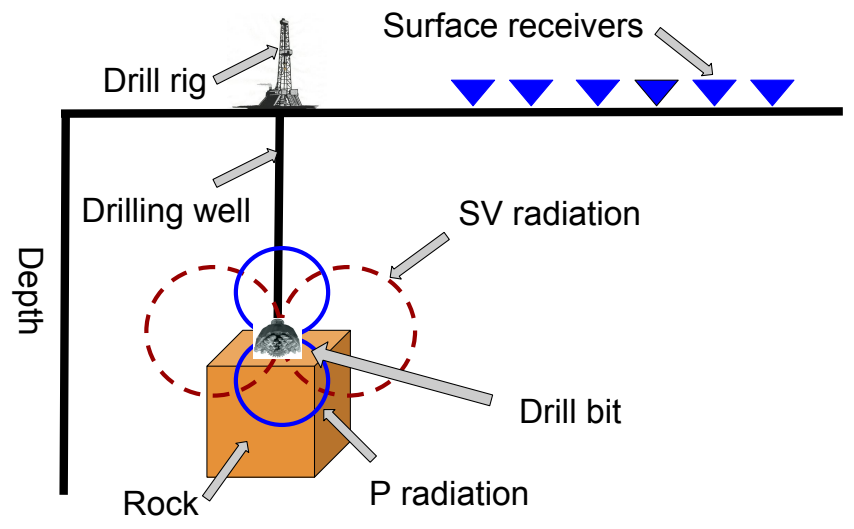

Fig. 2. Seismic-while-drilling radiation patterns.

\section{A. SWD radiation patterns}

While drilling, the drill-bit rock interaction radiates significant elastic, P- and S-wave, energy. In isotropic and homogeneous media, these radiations are functions of the drill-bit point force and the seismic velocities of rocks. It has been shown in [37], that for a roller-cone drill-bit, the seismic radiation pattern proceeding from the axial component drill-bit impacts can be modeled as a transient, monopolar point force acting along the axis of the borehole. Let us denote $U_{r}$ the Pwave radiation and $U_{\phi}$ the $\mathrm{S}$-wave motion. These radiations can be measured at the surface using the drill-bit seismic methods described above. More precisely, the far-field radial displacement resulting from the point force $w(t, L)$ satisfies the following relation

$$
U_{r}(r, \phi, t)=\frac{A_{1} \cos (\phi)}{\rho_{f} V_{p}^{2} r} w\left(t-\frac{r}{V_{p}}, L\right),
$$

and the far-field angular displacement satisfies

$$
U_{\phi}(r, \phi, t)=\frac{A_{1} \sin (\phi)}{\rho_{f} V_{s}^{2} r} w\left(t-\frac{r}{V_{s}}, L\right),
$$

where $r$ is the straight line distance from the source to the wavefront, $\rho_{f}$ is the rock density, $V_{p}$ is the rock compressional velocity, $V_{s}$ the rock shear velocity, $A_{1}$ is a constant, and the angle $\phi$ is measured relative to the direction of the point force (i.e. relative to the direction of axial drill-tooth impacts at the bottom of the borehole). The rock density $\rho_{f}$ can be expressed as a function of the rock compressional velocity $V_{p}$. More precisely, we have (see [38] for details)

$$
\rho_{f}=1.74 V_{p}^{0.25}
$$

where $V_{p}$ is expressed in km.s ${ }^{-1}$ and $\rho$ in g.cm ${ }^{-3}$.

\section{EXPRESSION OF THE DRILL-BIT AXIAL FORCE}

In this section, we express the axial force exerted at the drillbit as a (delayed) function of the topside velocity and hook load. This is done deriving the Riemann invariants associated to (1) and using a backstepping approach.

\section{A. Derivation of Riemann invariants}

The Riemann invariants of a hyperbolic PDE are the states corresponding to a transformation of the system which has a diagonalized transport matrix, i.e. the system can be written as a series of transport equations only coupled through the source terms [39]. On the set $\{(t, x) \mid \quad 0<t<T, \quad x \in[0, L]\}$, we define the Riemann invariants as

$$
\begin{aligned}
& u(t, x)=\frac{\partial}{\partial t} \xi(t, x)-c_{\xi} \frac{\partial}{\partial x} \xi(t, x), \\
& z(t, x)=\frac{\partial}{\partial t} \xi(t, x)+c_{\xi} \frac{\partial}{\partial x} \xi(t, x) .
\end{aligned}
$$

This transformation enables us to rewrite (1) as the PDE system

$$
\begin{aligned}
\frac{\partial}{\partial t} u(t, x)+c_{\xi} \frac{\partial}{\partial x} u(t, x) & =-\frac{k_{\xi}}{2}(u(t, \xi)+z(t, \xi)), \\
\frac{\partial}{\partial t} z(t, x)-c_{\xi} \frac{\partial}{\partial x} z(t, x) & =-\frac{k_{\xi}}{2}(u(t, \xi)+z(t, \xi)) .
\end{aligned}
$$

The boundary condition (5) rewrites

$$
u(t, 0)=z(t, 0)+\frac{2 c_{\xi}}{E A} w_{0}(t),
$$

while the boundary condition (8) rewrites

$$
\begin{aligned}
z(t, L)= & -u(t, L)+2 \dot{X}(t) \\
\ddot{X}(t)= & -\frac{a \zeta \epsilon}{M_{b} \omega_{\mathrm{bit}}} \dot{X}(t)-\frac{w_{f}}{M_{b}} \\
& -\frac{E A}{2 c_{\xi} M_{b}}(z(t, L)-u(t, L)) .
\end{aligned}
$$

\section{B. A backstepping transformation}

In this section, we use a backstepping transformation [40] to map the system (14)-(15) into a diagonal target system. This method consists in performing an integral change of variables (usually using a Volterra transformation) that maps the original system to a so-called "target system" for which the analysis is easier. Provided that the transformation is invertible (which is always the case for a Volterra transformation [40]), the original system and the corresponding target system have equivalent properties [40]. Let us first consider the following change of coordinates

$$
\hat{u}(t, x)=u(t, x) \mathrm{e}^{\frac{k_{\xi}}{2 c_{\xi}} x}, \quad \hat{z}(t, x)=z(t, x) \mathrm{e}^{-\frac{k_{\xi}}{2 c_{\xi}} x},
$$


so that the system (14)-(15) rewrites

$$
\begin{aligned}
\frac{\partial}{\partial t} \hat{u}(t, x)+c_{\xi} \frac{\partial}{\partial x} \hat{u}(t, x) & =-\frac{k_{\xi}}{2} \mathrm{e}^{\frac{k_{\xi}}{c_{\xi}} x} \hat{z}(t, x), \\
\frac{\partial}{\partial t} \hat{z}(t, x)-c_{\xi} \frac{\partial}{\partial x} \hat{z}(t, x) & =-\frac{k_{\xi}}{2} \mathrm{e}^{-\frac{k_{\xi}}{c_{\xi}} x} \hat{u}(t, x) .
\end{aligned}
$$

We consider the following Volterra transformation which is adjusted from the one defined in [41], [42]

$$
\begin{aligned}
\alpha(t, x)=\hat{u}(t, x) & +\int_{0}^{x}\left(K^{u u}(x, y) \hat{u}(t, y)\right. \\
& \left.+K^{u z}(x, y) \hat{z}(t, y)\right) d y \\
\beta(t, x)=\hat{z}(t, x) & +\int_{0}^{x}\left(K^{z u}(x, y) \hat{u}(t, y)\right. \\
& \left.+K^{z z}(x, y) \hat{z}(t, y)\right) d y
\end{aligned}
$$

where the kernels $K^{u u}, K^{z u}, K^{u z}$ and $K^{z z}$ are continuous functions defined on the domain $\mathcal{T}=\left\{(x, y) \in[0, L]^{2}, y \leq\right.$ $x\}$. They are defined by the following set of PDEs

$$
\begin{aligned}
\frac{\partial}{\partial x} K^{u u}(x, y)+\frac{\partial}{\partial y} K^{u u}(x, y) & =\frac{k_{\xi}}{2 c_{\xi}} \mathrm{e}^{-\frac{k_{\xi}}{c_{\xi}} y} K^{u z}, \\
\frac{\partial}{\partial x} K^{u z}(x, y)-\frac{\partial}{\partial y} K^{u z}(x, y) & =\frac{k_{\xi}}{2 c_{\xi}} \mathrm{e}^{\frac{k_{\xi}}{c_{\xi}} y} K^{u u}, \\
\frac{\partial}{\partial x} K^{z u}(x, y)-\frac{\partial}{\partial y} K^{z u}(x, y) & =-\frac{k_{\xi}}{2 c_{\xi}} \mathrm{e}^{-\frac{k_{\xi}}{c_{\xi}} y} K^{z z}, \\
\frac{\partial}{\partial x} K^{z z}(x, y)+\frac{\partial}{\partial y} K^{z z}(x, y) & =-\frac{k_{\xi}}{2 c_{\xi}} \mathrm{e}^{\frac{k_{\xi}}{c_{\xi}} y} K^{z u},
\end{aligned}
$$

with the boundary conditions

$$
\begin{aligned}
& K^{u u}(x, 0)=0, \quad K^{u z}(x, x)=\frac{k_{\xi}}{4 c_{\xi}} \mathrm{e}^{\frac{k_{\xi}}{c_{\xi}} x} \\
& K^{z z}(x, 0)=0, \quad K^{z u}(x, x)=-\frac{k_{\xi}}{4 c_{\xi}} \mathrm{e}^{-\frac{k_{\xi}}{c_{\xi}} x} .
\end{aligned}
$$

It is shown that the system (24)-(29) admits a unique continuous solution on $\mathcal{T}$ [41]. The proof is quite classical (see [43], [44] and [45]) and consists in writing the integral equations associated to equations (24)-(29). These integral equations are then solved using the method of successive approximations [40]. It is also possible to explicitly solve equations (24)-(29) (see [46] for details). For all $(x, y) \in \mathcal{T}$, we have

$$
K^{u u}(x, y)=\frac{k_{\xi}}{2 c_{\xi}} \mathrm{e}^{\frac{k_{\xi}(x-y)}{2 c_{\xi}}} \frac{y I_{1}\left(\sqrt{x^{2}-y^{2}} \frac{k_{\xi}}{2 c_{\xi}}\right)}{\sqrt{x^{2}-y^{2}}},
$$

where $I_{1}$ is the modified Bessel function of first kind. The kernel $K^{u v}$ can be obtained using equations (25). Finally the remaining kernels can be found by changing $k_{\xi}$ in $-k_{\xi}$.

Differentiating (22)-(23) with respect to time and space and integrating by parts, one can show that the states $\alpha$ and $\beta$ satisfy the following set of transport PDEs

$$
\begin{aligned}
\frac{\partial}{\partial t} \alpha(t, x)+c_{\xi} \frac{\partial}{\partial x} \alpha(t, x) & =-c_{\xi} K^{u z}(x, 0) \beta(t, 0), \\
\frac{\partial}{\partial t} \beta(t, x)-c_{\xi} \frac{\partial}{\partial x} \beta(t, x) & =c_{\xi} K^{z u}(x, 0) \alpha(t, 0) .
\end{aligned}
$$

with the topside boundary condition

$$
\alpha(t, 0)=\beta(t, 0)+\frac{2 c_{\xi}}{E A} w_{0}(t) .
$$

We choose not to write the boundary condition at $x=L$ as this one is not required in the following.

As the transformation (22)-(23) is a Volterra transformation, it is invertible [40]. The inverse transformation is defined by

$$
\begin{aligned}
\hat{u}(t, x)=\alpha(t, x) & +\int_{0}^{x}\left(L^{\alpha \alpha}(x, y) \alpha(t, y)\right. \\
& \left.+L^{\alpha \beta}(x, y) \beta(t, y)\right) d y \\
\hat{z}(t, x)=\beta(t, x) & +\int_{0}^{x}\left(L^{\beta \alpha}(x, y) \alpha(t, y)\right. \\
& \left.+L^{\beta \beta}(x, y) \beta(t, y)\right) d y
\end{aligned}
$$

where the kernels $L^{\alpha \alpha}, L^{\beta \alpha}, L^{\alpha \beta}$ and $L^{\beta \beta}$ are continuous functions defined on the domain $\mathcal{T}=\left\{(x, y) \in[0,1]^{2}, y \leq\right.$ $x\}$. They satisfy the following Volterra equation [40] defined for $0 \leq y \leq x \leq 1$.

$$
L(x, y)=-K(x, y)-\int_{\xi}^{x} K(x, \nu) L(\nu, y) d \nu
$$

where we have defined the functions $K(\cdot, \cdot)$ and $L(\cdot, \cdot)$ as

$$
\begin{aligned}
K(x, y) & =\left(\begin{array}{ll}
K^{u u}(x, y) & K^{u v}(x, y) \\
K^{v u}(x, y) & K^{v v}(x, y)
\end{array}\right), \\
L(x, y) & =\left(\begin{array}{ll}
L^{\alpha \alpha}(x, y) & L^{\alpha \beta}(x, y) \\
L^{\beta \alpha}(x, y) & L^{\beta \beta}(x, y)
\end{array}\right) .
\end{aligned}
$$

These inverse kernels are required to express the force exerted on the bit as a function of the topdrive force and velocity.

\section{A delayed equation}

In this section, we express for all $x \in[0, L]$ the Riemann invariants $\alpha(t, x)$ and $\beta(t, x)$ as functions of $\alpha(\cdot, 0)$ and $\beta(\cdot, 0)$. Then, using the inverse transformation (34)-(35), it becomes possible to express for all $x \in[0, L]$ the functions $u(t, x)$ and $z(t, x)$ as functions of $\alpha(\cdot, 0)$ and $\beta(\cdot, 0)$. Applying the method of characteristics on (31)-(32), for any $x \in[0, L]$ and for any $t \geq \frac{x}{c_{\xi}}$, we get

$$
\begin{aligned}
\alpha(t, x) & =\alpha\left(t-\frac{x}{c_{\xi}}, 0\right) \\
& -c_{\xi} \int_{0}^{\frac{x}{c_{\xi}}} K^{u z}\left(x-c_{\xi} s, 0\right) \beta(t-s, 0) d s, \\
\beta(t, x) & =\beta\left(t+\frac{x}{c_{\xi}}, 0\right) \\
& -c_{\xi} \int_{0}^{\frac{x}{c_{\xi}}} K^{z u}\left(x-c_{\xi} s, 0\right) \alpha(t+s, 0) d s .
\end{aligned}
$$


Combining these equations with (34), we obtain for all $t \geq \frac{L}{c_{\xi}}$

$$
\begin{aligned}
& \hat{u}(t, L)=\alpha\left(t-\frac{L}{c_{\xi}}, 0\right) \\
& -c_{\xi} \int_{0}^{\frac{L}{c_{\xi}}} K^{u z}\left(L-c_{\xi} s, 0\right) \beta(t-s, 0) d s \\
& +\int_{0}^{L} L^{\alpha \alpha}(L, y) \alpha\left(t-\frac{y}{c_{\xi}}, 0\right)+L^{\alpha \beta}(L, y) \beta\left(t+\frac{y}{c_{\xi}}, 0\right) d y \\
& -c_{\xi} \int_{0}^{L} \int_{0}^{\frac{y}{c_{\xi}}} L^{\alpha \alpha}(L, y) K^{u z}\left(y-c_{\xi} s, 0\right) \beta(t-s, 0) d s d y \\
& -c_{\xi} \int_{0}^{L} \int_{0}^{\frac{y}{c_{\xi}}} L^{\alpha \beta}(L, y) K^{z u}\left(y-c_{\xi} s, 0\right) \alpha(t+s, 0) d s d y .
\end{aligned}
$$

This implies, using Fubini's theorem and a simple change of variable

$$
\begin{aligned}
& \hat{u}(t, L)=\alpha\left(t-\frac{L}{c_{\xi}}, 0\right) \\
& -c_{\xi} \int_{0}^{\frac{L}{c_{\xi}}} K^{u z}\left(L-c_{\xi} s, 0\right) \beta(t-s, 0) d s \\
& +c_{\xi} \int_{0}^{\frac{L}{c_{\xi}}} L^{\alpha \alpha}\left(L, c_{\xi} y\right) \alpha(t-y, 0)+L^{\alpha \beta}\left(L, c_{\xi} y\right) \beta(t+y, 0) d y \\
& -c_{\xi} \int_{0}^{\frac{L}{c_{\xi}}} \int_{c_{\xi} s}^{L}\left(L^{\alpha \alpha}(L, y) K^{u z}\left(y-c_{\xi} s, 0\right) d y\right) \beta(t-s, 0) d s \\
& -c_{\xi} \int_{0}^{\frac{L}{c_{\xi}}} \int_{c_{\xi} s}^{L}\left(L^{\alpha \beta}(L, y) K^{z u}\left(y-c_{\xi} s, 0\right) d y\right) \alpha(t+s, 0) d s .
\end{aligned}
$$

Finally, using a new change of variable, we obtain

$$
\begin{aligned}
\hat{u}(t, L)=\alpha\left(t-\frac{L}{c_{\xi}}, 0\right) & +\int_{-\frac{L}{c_{\xi}}}^{\frac{L}{c_{\xi}}} c_{\xi} f_{1}^{u}(s) \alpha(t-s, 0) d s \\
& +\int_{-\frac{L}{c_{\xi}}}^{\frac{L}{c_{\xi}}} c_{\xi} f_{2}^{u}(s) \beta(t-s, 0) d s,
\end{aligned}
$$

where $f_{1}^{u}$ and $f_{2}^{u}$ are $L^{\infty}$ functions defined on the interval $\left[-\frac{L}{c_{\xi}}, \frac{L}{c_{\xi}}\right]$ by

$$
\begin{gathered}
f_{1}^{u}(s)=\mathbb{1}_{\left[0, \frac{L}{c_{\xi}}\right]}(s)\left(L^{\alpha \alpha}\left(L, c_{\xi} s\right)\right)-\mathbb{1}_{\left[-\frac{L}{c_{\xi}}, 0\right]}(s) \\
\left(\int_{-c_{\xi} s}^{L} L^{\alpha \beta}(L, y) K^{z u}\left(y+c_{\xi} s, 0\right) d y\right) \\
f_{2}^{u}(s)=\mathbb{1}_{\left[-\frac{L}{c_{\xi}}, 0\right]}(s)\left(L^{\alpha \beta}\left(L,-c_{\xi} s\right)\right)-\mathbb{1}_{\left[0, \frac{L}{c_{\xi}}\right]}(s)\left(K^{u z}(L-\right. \\
\left.\left.c_{\xi} s, 0\right)+\int_{c_{\xi} s}^{L} L^{\alpha \alpha}(L, y) K^{u z}\left(y-c_{\xi} s, 0\right) d y\right) .
\end{gathered}
$$

Similar computations can be done on the state $\hat{z}$ to obtain for all $t \geq \frac{L}{c_{\xi}}$

$$
\begin{gathered}
\hat{z}(t, L)=\beta\left(t+\frac{L}{c_{\xi}}, 0\right)+\int_{-\frac{L}{c_{\xi}}}^{\frac{L}{c_{\xi}}} c_{\xi} f_{1}^{z}(s) \alpha(t-s, 0) d s \\
+\int_{-\frac{L}{c_{\xi}}}^{\frac{L}{c_{\xi}}} c_{\xi} f_{2}^{z}(s) \beta(t-s, 0) d s,
\end{gathered}
$$

where $f_{1}^{z}$ and $f_{2}^{z}$ are $L^{\infty}$ functions defined on the interval $\left[-\frac{L}{c_{\xi}}, \frac{L}{c_{\xi}}\right]$ by

$$
\begin{aligned}
f_{1}^{z}(s) & =\mathbb{1}_{\left[0, \frac{L}{c_{\xi}}\right]}(s)\left(L^{\beta \alpha}\left(L, c_{\xi} s\right)\right)-\mathbb{1}_{\left[-\frac{L}{c_{\xi}}, 0\right]}(s)\left(\int_{-c_{\xi} s}^{L} L^{\beta \beta}(L, y)\right. \\
& \left.\cdot K^{z u}\left(y+c_{\xi} s, 0\right) d y+K^{z u}\left(L+c_{\xi} s, 0\right)\right) \\
f_{2}^{z}(s)= & \mathbb{1}_{\left[-\frac{L}{c_{\xi}}, 0\right]}(s)\left(L^{\beta \beta}\left(L,-c_{\xi} s\right)\right)-\mathbb{1}_{\left[0, \frac{L}{c_{\xi}}\right]}(s) \\
& \cdot\left(\int_{c_{\xi} s}^{L} L^{\beta \alpha}(L, y) K^{u z}\left(y-c_{\xi} s, 0\right) d y\right)
\end{aligned}
$$

Using these expressions we are now able to express the axial force on the drill-bit as a function of the top-drive force and velocity.

\section{Expression of the weight-on-bit}

Our objective is now to express the axial force at the drillbit as a function of the topside velocity and hook load. We have

$$
\begin{aligned}
w(t, L) & =-E A \frac{\partial \xi(t, L)}{\partial x}=E A \frac{u(t, L)-z(t, L)}{2 c_{\xi}} \\
& =E A \frac{\mathrm{e}^{-\frac{k_{\xi} L}{2 c_{\xi}}} \hat{u}(t, L)-\mathrm{e}^{\frac{k_{\xi} L}{2 c_{\xi}}} \hat{z}(t, L)}{2 c_{\xi}} .
\end{aligned}
$$

Using equations (39) and (42), and noticing that $\alpha(\cdot, 0)=$ $u(\cdot, 0)$ and $\beta(\cdot, 0)=z(\cdot, 0)$, we obtain

$$
\begin{aligned}
& w(t, L)=E A \frac{\mathrm{e}^{-\frac{k_{\xi} L}{2 c_{\xi}}} u\left(t-\frac{L}{c_{\xi}}, 0\right)-\mathrm{e}^{\frac{k_{\xi} L}{2 c_{\xi}}} z\left(t+\frac{L}{c_{\xi}}, 0\right)}{2 c_{\xi}} \\
& +\int_{-\frac{L}{c_{\xi}}}^{\frac{L}{c_{\xi}}}\left(f_{u}(s) u(t-s, 0)+f_{z}(s) z(t-s, 0) d y\right)
\end{aligned}
$$

where the functions $f_{u}$ and $f_{z}$ are respectively defined by

$$
\begin{aligned}
f_{u}(s) & =\frac{E A}{2}\left(\mathrm{e}^{-\frac{k_{\xi} L}{2 c \xi}} f_{1}^{u}(s)-\mathrm{e}^{\frac{k_{\xi} L}{2 c_{\xi}}} f_{1}^{z}(s)\right) \\
f_{z}(s) & =\frac{E A}{2}\left(\mathrm{e}^{-\frac{k_{\xi} L}{2 c_{\xi}}} f_{2}^{u}(s)-\mathrm{e}^{\frac{k_{\xi} L}{2 c_{\xi}}} f_{2}^{z}(s)\right) .
\end{aligned}
$$

Using the expressions of the Riemann invariants $u(t, x)$ and $z(t, x)$ given in (12)-(13) and the definition of the axial force and velocity, we immediately obtain

$$
\begin{aligned}
w(t, L)= & \frac{E A}{2 c_{\xi}}\left(\mathrm{e}^{-\frac{k_{\xi} L}{2 c_{\xi}}} v\left(t-\frac{L}{c_{\xi}}, 0\right)-\mathrm{e}^{\frac{k_{\xi} L}{2 c_{\xi}}} v\left(t+\frac{L}{c_{\xi}}, 0\right)\right) \\
& +\frac{1}{2}\left(\mathrm{e}^{-\frac{k_{\xi} L}{2 c_{\xi}}} w\left(t-\frac{L}{c_{\xi}}, 0\right)+\mathrm{e}^{\frac{k_{\xi} L}{2 c_{\xi}}} w\left(t+\frac{L}{c_{\xi}}, 0\right)\right) \\
& +\int_{-\frac{L}{c_{\xi}}}^{\frac{L}{c_{\xi}}}\left(f_{u}(s)+f_{z}(s)\right) v(t-s, 0) d s \\
& +\int_{-\frac{L}{c_{\xi}}}^{\frac{L}{c_{\xi}}} \frac{c_{\xi}}{E A}\left(f_{u}(s)-f_{z}(s)\right) w(t-s, 0) d s .
\end{aligned}
$$

It is interesting to note that this expression does not directly depend on the downhole boundary condition. In the next section, we describe a procedure to use this relation to estimate the compressional and shear velocities. 


\section{ROCK VELOCITY ESTIMATION}

Considering a single channel sensor, the radial component of the far-field recorded seismic-while-drilling data $\mathbf{d}^{\text {obs }}=$ $U_{r}^{o b s}(r, \phi, t)$ can be modelled by using equations (9) and (11) as

$$
\mathbf{d}^{c a l}=U_{r}^{c a l}(r, \phi, t)=\frac{A_{1} \cos (\phi)}{1.74 V_{p}^{0.25} V_{p}^{2} r} w\left(t-\frac{r}{V_{p}}, L\right),
$$

where $w$ is the calculated force-on-bit with equation (49). As clear, the recorded data is the delayed and amplitude modulated version of the force-on-bit. Hence, in noise free data, simple cross-correlation of the calculated force-on-bit with the recorded data can provide a rough estimate of the velocity. In other words, by measuring the similarity between the recorded data and the function $w$, the estimated lag, i.e., $\frac{r}{V_{p}}$, between the two data sets gives a rough estimate of the rock velocity $V_{p}$. In the case of noisy data, assuming that the data is contaminated with white Gaussian noise, we propose to solve

$$
\left\{\hat{A}_{1}, \hat{V}_{p}\right\}=\underset{A_{1}, V_{p}}{\operatorname{argmin}}\left\|\mathbf{d}^{o b s}-\mathbf{d}^{c a l}\right\|_{2}^{2} .
$$

The optimization problem of equation (51) can be rewritten as

$$
\left\{\hat{A}_{1}, \hat{V}_{p}\right\}=\underset{A_{1}, V_{p}}{\operatorname{argmin}} \sum_{i=1}^{N}\left(U_{r}^{o b s}\left(r, \phi, t_{i}\right)-U_{r}^{c a l}\left(r, \phi, t_{i}\right)\right)^{2},
$$

where $N$ is the number of time samples in the data. It is worth mentioning that equation (52) is only valid for direct arrival of the primary wave in the case of single isotropic and homogeneous rock. However, the SWD data contains different wave modes. In dealing with such dataset, to estimate the velocity of rock interacting with the drill-bit, similar to the work of Rector and Hardage [37] we should match the amplitude of the direct arrival in the source compensated SWD dataset with the amplitude of the modeled radiation pattern of the direct arrival given by equation (50). Nonetheless, source estimation is a necessary step in both cases. In this paper, we consider a single isotropic and homogenous rock simulation so the only wavefield generated is the direct arrival. Accordingly, we use equation (52) for the rock velocity estimation process. The cost function of equation (52) can be solved with alternating minimization algorithm (Algorithm 1). As the alternating minimization method is a local minimization technique, we use the rough velocity estimated by the cross-correlation approach as an initial solution. Steps 1 and 2 in the algorithm is solved with least-squares parameter estimation technique. Note that the proposed approach can be adjusted to estimate the shear velocity $V_{s}$ using (10).

\section{Simulation Results}

In this section, we evaluate through simulations the performance of the estimation of the weight-on-bit obtained with equation (49) in the presence of noisy measurements. Then, we analyze the performance of the rock velocity estimation algorithm designed in Section V (i.e., Algorithm 1). We test the efficiency of the algorithm on single channel synthetic data, and then, extend the approach to a multichannel recording. $\overline{\text { Algorithm } 1 \text { Alternating minimization algorithm for rock }}$ velocity estimation

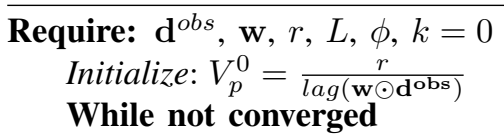

1: $\quad$ Update Amplitude

$$
\begin{array}{ll} 
& A_{1}^{k+1}=\underset{A_{1}}{\operatorname{argmin}} \sum_{i=1}^{N}\left(U_{r}^{o b s}\left(r, \phi, t_{i}\right)\right. \\
\text { 2: } & \left.\frac{A_{1} \cos (\phi)}{1.74\left(V_{p}^{k}\right)^{0.25}\left(V_{p}^{k}\right)^{2} r} w\left(t_{i}-\frac{r}{V_{p}^{k}}, L\right)\right) \\
\quad \text { Update Velocity } & V_{p}^{k+1}=\underset{V_{p}}{\operatorname{argmin}} \sum_{i=1}^{N}\left(U_{r}^{o b s}\left(r, \phi, t_{i}\right)-\frac{A_{1}^{k+1} \cos (\phi)}{1.74 V_{p}^{0.25} V_{p}^{2} r} w\left(t_{i}-\right.\right. \\
\text { 3: } & \left.\left.\frac{r}{V_{p}}, L\right)\right) \quad \text { Update } k \leftarrow k+1 \\
& \text { If converged } \\
& \text { Output } \\
& \hat{V}_{p} \leftarrow V_{p}^{k} \\
\hat{A}_{1} \leftarrow A_{1}^{k}
\end{array}
$$

\begin{tabular}{|c|c||c|c|}
\hline Param. & Value & Param. & Value \\
\hline \hline$E$ & $2 \times 10^{11} \mathrm{~Pa}$ & $\rho$ & $8000 \mathrm{~kg} \cdot \mathrm{m}^{-3}$ \\
$c_{\xi}$ & $5000{\mathrm{~m} . \mathrm{s}^{-1}}^{-3}$ & $k_{\xi}$ & $0.3 \mathrm{~s}^{-1}$ \\
$A$ & $3.5 \times 10^{-3} \mathrm{~m}^{2}$ & $L$ & $2000 \mathrm{~m}$ \\
$M_{b}$ & $12000 \mathrm{~kg}$ & $\omega_{b i t}$ & $1.05 \mathrm{~s}^{-1}$ \\
$a$ & $0.1 \mathrm{~m}$ & $\zeta$ & 0.6 \\
$w_{f}$ & $71280 \mathrm{~N}$ & $\phi$ & $0.1745 \mathrm{rad}$ \\
\hline \multicolumn{4}{|c}{ TABLE II }
\end{tabular}

NUMERICAL VALUES OF THE PARAMETERS

\section{A. Estimation of the drill-bit source}

Consider a drill-string whose axial motion satisfies the PDE given by equation (1) subject to the boundary conditions given in equations (5)-(8). Table II shows the numerical values of parameters for the drill-string system. The drill-bit is interacting with a sedimentary rock $\left(\epsilon=57 \mathrm{Jcm}^{-3}\right)$. The PDE system is simulated using a classical finite volume method based on a Godunov scheme [39]. The control input $w_{0}$ is chosen as a sinusoidal function. The corresponding force-onbit is denoted $F_{b i t}^{r e a l}$. By using the top-drive velocity and force measurements and equation (49), we can estimate the force-on-bit $F_{b i t}^{e s t}$. To do so, first, we need to compute the kernels $K^{*}$. A numerical solution to equations (24)-(25) can be reached with the method of characteristics and a fixed point algorithm (see [47] for details) or the explicit expression given by equation (30). The different integrals are computed using a trapezoidal method with adjustable precision. We consider the case of noisy measurements, the noise is modeled by a white Gaussian noise that is characterized by its signal-to-noise ratio (SNR). More precisely, similar to what is done in [48], we define the SNR as

$$
S N R=\frac{a_{r m s}^{2}}{\sigma_{n}^{2}},
$$

where $a_{r m s}$ is the root-mean-square amplitude of the noisefree signal, and $\sigma_{n}^{2}$ is the variance of the noise. Choosing a value for the SNR gives the corresponding amplitude for the 
white Gaussian noise. Note that the value of the SNR may be different for the velocity and force measurements.

The estimated force-on-bit $F_{\text {bit }}^{e s t}$ is compared with the ground truth $F_{b i t}^{\text {real }}$ in Figure 3 . We use the sedimentary rock and noisy top-drive measurements with $S N R=5$. The bit is not rotating, only bouncing axially. We notice that after a time $\frac{L}{c_{\xi}}$, the functions are comparable (they are not identical due to the presence of the noise). Note that the estimated force-on-bit is not estimated for $t \leq \frac{L}{c_{\xi}}$ or $t \geq T_{f}-\frac{L}{c_{\xi}}$ (where $T_{f}$ is the final time of the simulation) due to the fact that, on these intervals, we cannot compute all terms present in equation (49).

As is done in [48], to examine the performance of the proposed method, we introduce the quality of the reconstruction metric for the estimated force-on-bit. Let us denote $y_{0}$ as true generic signal (in our case $y_{0}=F_{b i t}^{r e a l}$ ) and let us denote by $y$ its estimation (in our case $y=F_{b i t}^{e s t}$ ). We define the quality of the reconstruction $Q$ as follows

$$
Q=10 \log \frac{\left\|y_{0}\right\|_{2}^{2}}{\left\|y_{0}-y\right\|_{2}^{2}},
$$

where $\|\cdot\|_{2}$ is the $\ell_{2}$ norm. Figure 4 shows the variation of $Q$ for different values of the SNR of top-drive velocity and the top-drive force (the SNR being chosen between 0.5 and 10). We have also plotted a dashed line that corresponds to the limit value of $Q=10$. We have a symmetrical effect between the SNR of top-drive velocity and the SNR of top-drive force. Moreover, the critical value of $Q=10$ is not reached for low SNR values $(S N R<2)$. In our simulations, we find that when the quality factor is larger than 10 , the rock velocity estimation algorithm results in satisfactory performances, even in the presence of severe noise in the SWD data. It is worth noting that the noise level in top-drive measurements is different from the noise level in the SWD data. Next, we evaluate the performance of the rock velocity estimation algorithm on single and multichannel SWD datasets.

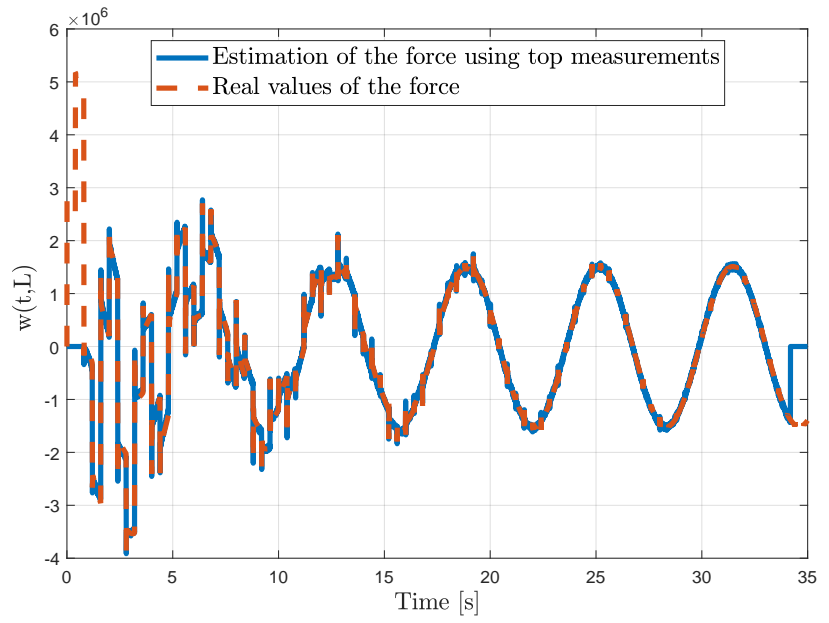

Fig. 3. Comparison of the simulated force-on-bit and of the estimated one (computed using (49)) using noisy top-drive measurements in the case of sedimentary rock $(\mathrm{SNR}=5)$.

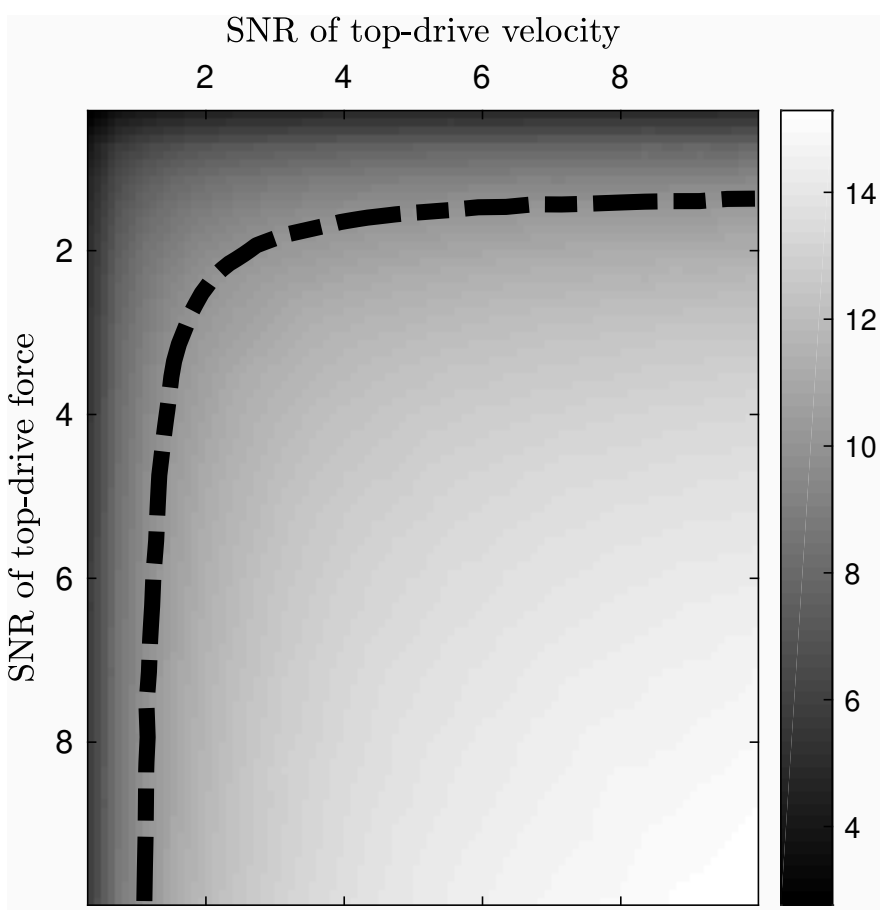

Fig. 4. Sensitivity analysis of the drill-bit source estimation to different levels of noise in the top-drive measurements. We use 75 realizations for each noise level combination and report the average value of the quality of the reconstruction $Q$. The dashed line corresponds to $Q=10$ contour which we consider it to be the success limit for the drill-bit source estimation.

\section{B. Single sensor}

We now use Algorithm (1) to estimate the velocity of the rocks that are interacting with the drill-bit. Let us consider that only one seismic sensor is available with its location being characterized by the angle $\phi$ and the distance $r=\frac{L}{\cos (\phi)}$ (see Section III for details). The numerical values of the different parameters corresponding to the drill-string system are given in Table II. We analyse the efficiency of the proposed algorithm on three different types of rocks with different hardness, i.e., different compressional/shear velocities,

- Unconsolidated sands (i.e. water saturated) for which $V_{p}=1750 \mathrm{~m} . \mathrm{s}^{-1}, \rho_{f}=2 \mathrm{~g} . \mathrm{cm}^{-3}$ and $\epsilon=11$ $\mathrm{J} . \mathrm{cm}^{-3}$.

- Sedimentary rocks (e.g. sandstone) for which $V_{p}=3500$ $\mathrm{m} . \mathrm{s}^{-1}, \rho_{f}=2.4 \mathrm{~g} . \mathrm{cm}^{-3}$ and $\epsilon=57 \mathrm{~J} . \mathrm{cm}^{-3}$.

- Igneous or metamorphic rocks (e.g. granite) for which $V_{p}=5750 \mathrm{~m} . \mathrm{s}^{-1}, \rho_{f}=2.7 \mathrm{~g} . \mathrm{cm}^{-3}$ and $\epsilon=151$ J.cm ${ }^{-3}$.

Using the simulated weight-on-bit and the (known) value of $V_{p}$, we compute the function $U_{r}^{o b s}$ to which we add white Gaussian noise with SNR equal to 10. Moreover, by using the top-drive force and velocity measurements, which are given by the simulation of the PDE, we estimate the weight-on-bit (see equation (49)). We have plotted in Figure 5, the time evolution of the far-field radial displacement provided by almost noisefree seismic sensors for different kind of rocks (unconsolidated sands, sedimentary rocks, metamorphic rocks). The angle $\phi$ is equal to 10 degrees.

Let us test our approach in the case of unconsolidated sands. 


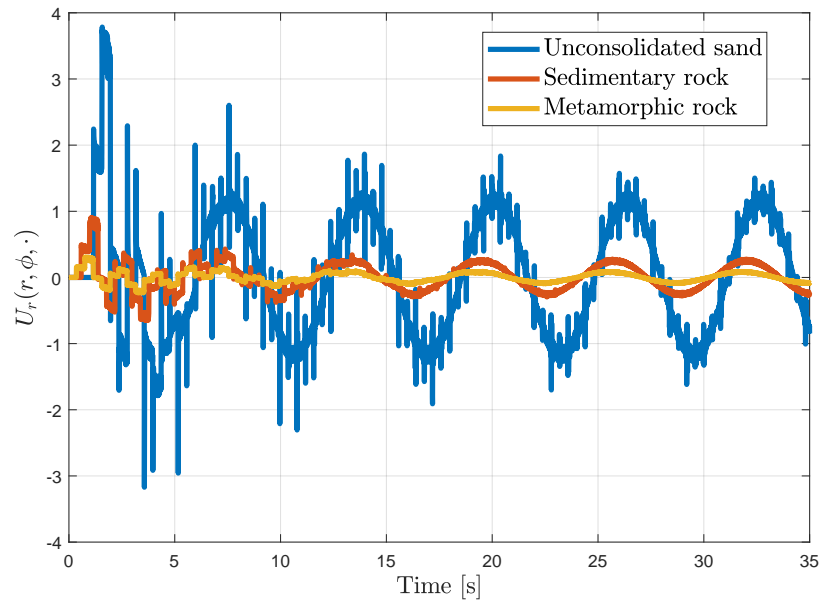

Fig. 5. Comparison of the far-field radial displacement $U_{r}(\phi=10$ degrees $)$ with respect to time for different rocks (unconsolidated sands, sedimentary rocks, metamorphic rocks).

We have plotted in Figure 6 the cross correlation between $U_{r}$ and $w$. The largest spike occurs at $t_{a}=1.155 \mathrm{~s}$ which gives us the corresponding estimate

$$
V_{p}^{0}=\frac{r}{t_{a}}=\frac{L}{t_{a} \cos (\phi)}=1758 \mathrm{~ms}^{-1},
$$

which is extremely close to the real value $\left(1750 \mathrm{~ms}^{-1}\right)$. Finally, for each type of rock, we give in Table III the different

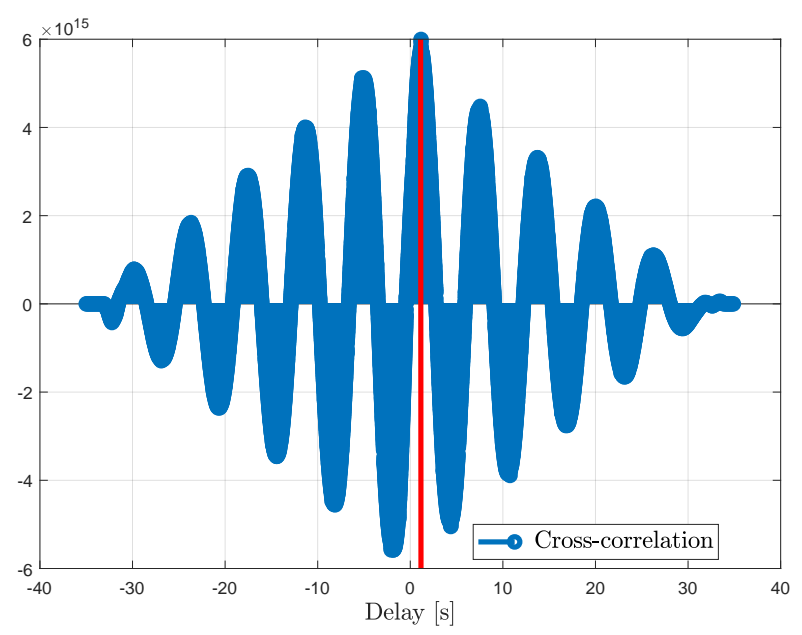

Fig. 6. Cross correlation between $\mathbf{d}^{\text {obs }}$ and $\mathbf{w}$ for unconsolidated sands, the largest spike occurs at $t=1.155 \mathrm{~s}(\mathrm{SNR}=10)$.

estimates for $V_{p}$ obtained using our Algorithm. We compare these results with the ones obtained using only the crosscorrelation estimate. One can notice that even if they are extremely similar, the proposed algorithm slightly improves the quality of the estimations.

\section{Multiple sensors}

Let us now consider that several sensors are available. For each sensor, the procedure described in the previous section can still be applied. Then, we can compute the mean and

\begin{tabular}{|c|c|c|c|}
\hline Rock & Ground truth $V_{p}\left(\mathrm{~ms}^{-1}\right)$ & $V_{p}^{\mathrm{D}}$ & $V_{p}^{10}$ \\
\hline \hline Unc. sands & 1750 & 1758 & 1751 \\
Sedimentary & 3500 & 3563 & 3489 \\
Metamorphic & 5750 & 5875 & 5774 \\
\hline \multicolumn{3}{|c|}{ TABLE III }
\end{tabular}

ESTIMATION OF THE COMPRESSIONAL VELOCITY $\alpha$ USING A CROSS-CORRELATION $\left(V_{p}^{0}\right)$ AND ALGORITHM 1 (10 ITERATIONS). THE SNR FOR THE SIGNAL $U_{r}$ IS EQUAL TO 10.

\begin{tabular}{|c|c|c|c|c|}
\hline & SNR & 0.1 & 0.5 & 1 \\
\hline \hline Unconsolidated & Mean & 1711 & 1753 & 1753 \\
Sands & Standard Deviation & 58 & 26 & 20 \\
\hline Sedimentary & Mean & 3480 & 3496 & 3498 \\
Rocks & Standard Deviation & 60 & 38 & 27 \\
\hline Metamorphic & Mean & 5715 & 5741 & 5746 \\
Rocks & Standard Deviation & 106 & 74 & 72 \\
\hline
\end{tabular}

MEAN AND STANDARD DEVIATION OF THE OBTAINED ESTIMATIONS COMPARED TO THE REAL VELOCITY FOR DIFFERENT SNR FOR DIFFERENT TYPES OF ROCKS (100 SENSORS).

the standard deviations of the obtained set of data. Table IV shows the mean and the standard deviation of the obtained estimations for different SNR for the three different kinds of rocks using 100 different sensors. One can check that even in the presence of strong noise ( $\mathrm{SNR}=0.1$ ), the mean remains close to the real value while the standard deviation remains small. This highlights the quality of the proposed approach. Our results indicate that the drilling process with a pure axial motion can provide a better estimation of the velocities in the case of unconsolidated rocks, i.e., near surface, than the hard rocks. Moreover, as the SNR decreases the performance of the method is deteriorated.

\section{FUTURE WORK}

In this paper, we focused on the axial motion of the drill-bit. However, the torsional motion of the drill-bit also contributes to the overall signature of the drill-bit source. To properly model and estimate the elastic source signature both axial and torsional motions should be incorporated in the estimation system. Moreover, the main focus of the proposed algorithm was to provide a good estimate of the drill-bit source signature and to invert the direct arrival for the velocity of the rocks that are interacting with the drill-bit. Rector and Hardage [37] show that the radiation patterns for the direct arrival in the field data, after removing the source signature and compensating for the quality factor of the earth, can be approximated by equation (9). However, the SWD data contains a variety of wave modes such as up-going and down-going reflected wave fields, converted waves, head waves, rig arrivals, and so on. To properly see ahead of the drill-bit and monitor the rock properties, the full waveform should be considered and inverted. Recently, the authors proposed an imaging workflow for SWD data [49], [50]. The results show that if the drill-bit source signature is known, the pre-stack depth imaging of such dataset is achievable. Moreover, authors also combined the SWD data with the surface seismic dataset and jointly image the subsurface [30]. Accordingly, the approach presented in this paper for estimating the drill-bit source signature can be 
used as an input to the SWD imaging workflows. The fullwaveform inversion (FWI) is also possible. FWI is an approach that directly inverts the full recorded seismic wavefield for the rock velocities of the subsurface [51]. As the FWI algorithm is highly nonlinear, the initial estimate of the rock velocity is an important factor for preventing the solution to be trapped in local minima. The source signature is also one of the input parameters of the conventional FWI method. The background velocity information usually is borrowed from the kinematics analysis of the surface seismic data. Hence, FWI inversion of the SWD dataset, after estimating the drill-bit source signature and using the background velocity information, is possible. In the case of SWD data, the FWI inversion result of surface seismic data could also be used as an initial estimate. Note that the background velocity information or the velocity calculated within surface seismic FWI can also be used as an initial estimate of the rock velocity that is interacting with the drillbit (i.e., initial velocity in Algorithm 1). The full-waveform inversion of the SWD dataset, in theory, can provide the subsurface velocity around and ahead of the drill-bit. To date, we are not aware of any developed FWI algorithm for SWD measurements.

\section{CONCLUDING REMARKS}

We have derived a new algorithm that provides a near realtime estimation of the seismic velocities of rocks interacting with the drill-bit, using seismic-while-drilling and drillstring top-drive force and top-derive velocity measurements. The proposed algorithm combined a distributed representation of the drill-string dynamics, seismic-while-drilling measurements, and classical parameter estimations methods. The interesting feature of the presented methodology is that it does not require any explicit knowledge about the subsurface properties, in so far as only surface measurements are required. The approach provided an efficient and reliable estimation of the seismic velocities of rocks interacting with the drill-bit, enabling a more precise characterization of the subsurface. Integrating the velocity estimation with the existing control techniques can, in theory, lead to an improvement in the drilling performance by reducing non-productive time, increasing the ROP, and reducing the effect of undesired torsional oscillations. This is an important step towards a subsurfaceaware drilling system. Testing the algorithm against real-field data is the focus of our future contributions.

\section{ACKNOWLEDGMENTS}

This work was supported by the University of Calgary's Canada First Research Excellence Fund Program, the Global Research Initiative in Sustainable Low Carbon Unconventional Resources.

\section{REFERENCES}

[1] U. J. F. Aarsnes, F. Di Meglio, and R. J. Shor, "Avoiding stick slip vibrations in drilling through startup trajectory design," Journal of Process Control, vol. 70, pp. 24-35, oct 2018. [Online]. Available: https://linkinghub.elsevier.com/retrieve/pii/S0959152418301859

[2] U. J. F. Aarsnes, F. di Meglio, and R. Shor, "Benchmarking of industrial stick-slip mitigation controllers," IFAC-PapersOnLine, vol. 51, no. 8, pp. 233-238, 2018.
[3] M. Kapitaniak, V. V. Hamaneh, J. P. Chávez, K. Nandakumar, and M. Wiercigroch, "Unveiling complexity of drill-string vibrations: Experiments and modelling," International Journal of Mechanical Sciences, vol. 101, pp. 324-337, 2015.

[4] P. Spanos, A. Chevallier, N. P. Politis, and M. L. Payne, "Oil and gas well drilling: a vibrations perspective," The Shock and Vibration Digest, vol. 35, no. 2, pp. 85-103, 2003.

[5] F. Di Meglio and U. J. F. Aarsnes, "A distributed parameter systems view of control problems in drilling," IFAC-PapersOnLine, vol. 48, no. 6, pp. 272-278, 2015.

[6] B. Saldivar, S. Mondié, S.-I. Niculescu, H. Mounier, and I. Boussaada, "A control oriented guided tour in oilwell drilling vibration modeling," Annual Reviews in Control, vol. 42, pp. 100-113, 2016.

[7] I. Boussaada, H. Mounier, S.-I. Niculescu, and A. Cela, "Analysis of drilling vibrations: A time-delay system approach," in 2012 20th Mediterranean Conference on Control \& Automation (MED). IEEE, 2012, pp. 610-614.

[8] C. Germay, V. Denoël, and E. Detournay, "Multiple mode analysis of the self-excited vibrations of rotary drilling systems," Journal of Sound and Vibration, vol. 325, no. 1-2, pp. 362-381, aug 2009. [Online]. Available: http://linkinghub.elsevier.com/retrieve/pii/S0022460X09002478

[9] T. Richard, C. Germay, and E. Detournay, "Self-excited stickslip oscillations of drill bits," Comptes Rendus Mécanique, vol. 332, no. 8, pp. 619-626, aug 2004. [Online]. Available: http://linkinghub.elsevier.com/retrieve/pii/S1631072104001135

[10] V. A. Dunayevsky and F. Abbassian, "Application of Stability Approach to Bit Dynamics," SPE Drilling \& Completion, vol. 13, no. 2, pp. 22-25, jun 1998. [Online]. Available: https://www.onepetro.org/journalpaper/SPE-30478-PA

[11] J. D. Jansen, "Nonlinear dynamics of oilwell drillstrings," Ph.D. dissertation, Delft University of Technology, 1993. [Online]. Available: http://repository.tudelft.nl/assets/uuid:d205b287-bcdd-4b49b12a-963d9b906dea/3me_jansen_19930616.PDF

[12] B. Saldivar, S. Mondié, J.-J. Loiseau, and V. Rasvan, "Stick-slip oscillations in oillwell drilstrings: Distributed parameter and neutral type retarded model approaches," IFAC Proceedings Volumes (IFACPapersOnline), vol. 18, no. PART 1, pp. 284-289, 2011.

[13] P. C. Kriesels, W. . J. G. Keultjes, P. Dumont, I. Huneidi, O. Owoeye, R. A. Hartmann et al., "Cost savings through an integrated approach to drillstring vibration control," in SPE/IADC Middle East Drilling Technology Conference. Society of Petroleum Engineers, 1999.

[14] R. I. Leine, D. H. van Campen, and W. J. G. Keultjes, "Stick-slip Whirl Interaction in Drillstring Dynamics," Journal of Vibration and Acoustics, vol. 124, no. 2, p. 209, 2002. [Online]. Available: http://vibrationacoustics.asmedigitalcollection. asme.org/article. aspx?articleid=1470408

[15] K. Nandakumar and M. Wiercigroch, "Stability analysis of a state dependent delayed, coupled two DOF model of drill-string vibration," Journal of Sound and Vibration, vol. 332, no. 10, pp. 2575-2592, may 2013. [Online]. Available: http://linkinghub.elsevier.com/retrieve/pii/S0022460X1200987X

[16] J. F. Brett, "The Genesis of Bit-Induced Torsional Drillstring Vibrations," SPE Drilling Engineering, vol. 7, no. 03, pp. 168-174, sep 1992. [Online]. Available: http://www.onepetro.org/mslib/servlet/onepetropreview?id=00021943\&soc=SPE http://www.onepetro.org/doi/10.2118/21943-PA

[17] J. F. Brett, A. D. Beckett, C. A. Holt, and D. L. Smith, "Uses and Limitations of Drillstring Tension and Torque Models for Monitoring Hole Conditions," SPE Drilling Engineering, vol. 4, no. 03, pp. 223-229, sep 1989. [Online]. Available: http://www.onepetro.org/doi/10.2118/16664-PA

[18] G. W. Halsey, A. Kyllingstad, T. V. Aarrestad, and D. Lysne, "Drillstring Vibrations: Comparison Between Theory and Experiments on a FullScale Research Drilling Rig," in SPE/IADC Drilling Conference, no. IADC/SPE 14760. Society of Petroleum Engineers, apr 1986, pp. 311321. [Online]. Available: http://www.onepetro.org/doi/10.2118/14760MS

[19] D. Zhao, S. Hovda, and S. Sangesland, "Abnormal Down Hole Pressure Variation by Axial Stick-Slip of Drillstring," Journal of Petroleum Science and Engineering, vol. 145, pp. 194-204, 2016. [Online]. Available: http://dx.doi.org/10.1016/j.petrol.2016.04.004

[20] R. J. Shor, M. W. Dykstra, O. J. Hoffmann, and M. Coming, "For better or worse: applications of the transfer matrix approach for analyzing axial and torsional vibration," in SPE/IADC Drilling Conference and Exhibition. Society of Petroleum Engineers, 2015.

[21] R. Meehan, L. Nutt, N. Dutta, J. Menzies et al., "Drill bit seismic: A drilling optimization tool," in IADC/SPE drilling conference. Society of Petroleum Engineers, 1998. 
[22] F. Poletto and F. Miranda, Seismic while drilling: Fundamentals of drillbit seismic for exploration. Elsevier, 2004, vol. 35.

[23] A. Anchliya, "A review of seismic while drilling (swd) techniques: A journey from 1986 to 2005," in SPE Europec/EAGE Annual Conference and Exhibition. Society of Petroleum Engineers, 2006.

[24] R. Meehan, D. Miller, J. Haldorsen, M. Kamata, and B. Underhill, "Rekindling interest in seismic while drilling," Oilfield Review, vol. 5 , no. 1 , pp. 4-13, 1993.

[25] J. Greenberg, "Seismic while drilling keeps bit turning to right while acquiring key real-time data," Drilling Contractor, vol. 64, no. 2, pp. 44-45, 2008.

[26] G. Rossi, P. Corubolo, G. Böhm, P. Dell'Aversana, A. Vesnaver, F. Poletto, S. Morandi, and E. Ceragioli, "Joint 3d inversion of swd and surface seismic data," first break, vol. 19, no. 8, pp. 453-459, 2001.

[27] G. Bernasconi and M. Vassallo, "Efficient data compression for seismicwhile-drilling applications," IEEE transactions on geoscience and remote sensing, vol. 41, no. 3, pp. 687-696, 2003.

[28] I. Vasconcelos and R. Snieder, "Interferometry by deconvolution: Part 2-theory for elastic waves and application to drill-bit seismic imaging," Geophysics, vol. 73, no. 3, pp. S129-S141, 2008.

[29] H. Djikpesse, P. Armstrong, R. Rufino, and A. Hawthorn, "Reducing uncertainty with seismic measurements while drilling," IEEE Transactions on Instrumentation and Measurement, vol. 59, no. 1, pp. 4-14, 2010.

[30] N. Kazemi, R. Shor, and K. Innanen, "Illumination compensation with seismic-while-drilling plus surface seismic imaging," in 80th EAGE Conference and Exhibition 2018, 2018.

[31] P. Bharadwaj, L. Demanet, and A. Fournier, "Focused blind deconvolution," IEEE Transactions on Signal Processing, 2019.

[32] C. Germay, N. van de Wouw, H. Nijmeijer, and R. Sepulchre, "Nonlinear Drillstring Dynamics Analysis," SIAM Journal on Applied Dynamical Systems, vol. 8, no. 2, pp. 527-553, jan 2009. [Online]. Available: http://epubs.siam.org/doi/abs/10.1137/060675848

[33] E. Cayeux, "On the Importance of Boundary Conditions for Real-Time Transient Drill-String Mechanical Estimations," in IADC/SPE Drilling Conference and Exhibition. Society of Petroleum Engineers, mar 2018. [Online]. Available: http://www.onepetro.org/doi/10.2118/189642-MS

[34] U. J. F. Aarsnes and O. M. Aamo, "Linear stability analysis of self-excited vibrations in drilling using an infinite dimensional model," Journal of Sound and Vibration, vol. 360, pp. 239-259, jan 2016. [Online]. Available: http://linkinghub.elsevier.com/retrieve/pii/S0022460X15007385

[35] T. Richard, C. Germay, and E. Detournay, "A simplified model to explore the root cause of stick-slip vibrations in drilling systems with drag bits," Journal of Sound and Vibration, vol. 305, no. 3, pp. 432-456, aug 2007. [Online]. Available: http://linkinghub.elsevier.com/retrieve/pii/S0022460X07002908

[36] E. Detournay and P. Defourny, "A phenomenological model for the drilling action of drag bits," International Journal of Rock Mechanics and Mining Sciences \& Geomechanics Abstracts, vol. 29, no. 1, pp. 13-23, 1992. [Online]. Available: http://linkinghub.elsevier.com/retrieve/pii/0148906292910413

[37] J. Rector III and B. Hardage, "Radiation pattern and seismic waves generated by a working roller-cone drill bit," Geophysics, vol. 57, no. 10, pp. 1319-1333, 1992.

[38] T. M. Brocher, "Empirical relations between elastic wavespeeds and density in the earth's crust," Bulletin of the seismological Society of America, vol. 95, no. 6, pp. 2081-2092, 2005.

[39] R. J. LeVeque, Finite volume methods for hyperbolic problems. Cambridge university press, 2002.

[40] M. Krstic and A. Smyshlyaev, Boundary Control of PDEs: A Course on Backstepping Designs. SIAM, 2008.

[41] J.-M. Coron, R. Vazquez, M. Krstic, and G. Bastin, "Local Exponential $H^{2} \quad$ Stabilization of $\quad$ a $2 \quad$ x 2 Quasilinear Hyperbolic System Using Backstepping," SIAM Journal on Control and Optimization, vol. 51, no. 3, pp. 2005-2035, jan 2013. [Online]. Available: http://ieeexplore.ieee.org/document/6161075/ http://epubs.siam.org/doi/10.1137/120875739

[42] R. Vazquez, M. Krstic, and J.-M. Coron, "Backstepping boundary stabilization and state estimation of a 2 x 2 linear hyperbolic system," in IEEE Conference on Decision and Control and European Control Conference, vol. 1, no. 1. Ieee, dec 2011, pp. 49374942. [Online]. Available: http://ieeexplore.ieee.org/document/6160338/ http://ieeexplore.ieee.org/lpdocs/epic03/wrapper.htm?arnumber=6160338

[43] L. Hu, F. Di Meglio, R. Vazquez, and M. Krstic, "Control of Homodirectional and General Heterodirectional Linear Coupled
Hyperbolic PDEs," arXiv preprint, pp. 1-33, 2015. [Online]. Available: http://arxiv.org/abs/1504.07491

[44] F. John, "Continuous dependence on data for solutions of partial differential equations with a prescribed bound," Communications on pure and applied mathematics, vol. 13, no. 4, pp. 551-585, 1960.

[45] G. B. Whitham, Linear and nonlinear waves. John Wiley \& Sons, 2011, vol. 42.

[46] R. Vazquez and M. Krstic, "Marcum -functions and explicit kernels for stabilization of linear hyperbolic systems with constant coefficients," Systems \& Control Letters, vol. 68, no. 1, pp. 33-42, jun 2014. [Online]. Available: http://dx.doi.org/10.1016/j.sysconle.2014.02.008 http://linkinghub.elsevier.com/retrieve/pii/S0167691114000607

[47] J. Auriol, "Robust design of backstepping controllers for systems of linear hyperbolic pdes," Ph.D. dissertation, PSL Research University, 2018.

[48] N. Kazemi, E. Bongajum, and M. D. Sacchi, "Surface-consistent sparse multichannel blind deconvolution of seismic signals," IEEE Transactions on geoscience and remote sensing, vol. 54, no. 6, pp. 3200-3207, 2016.

[49] N. Kazemi, R. Shor, and K. Innanen, "Imaging with a seismic-whiledrilling dataset," CSPG CSEG CWLS Convention, 2018.

[50] N. Kazemi, D. Trad, K. Innanen, and R. Shor, "Least-squares rtm of a seismic-while-drilling dataset," arXiv preprint arXiv:1902.06889, 2019.

[51] J. Virieux and S. Operto, "An overview of full-waveform inversion in exploration geophysics," Geophysics, vol. 74, no. 6, pp. WCC1-WCC26, 2009. 